\title{
An Analysis on Gender Classification and Age Estimation Approaches
}

\author{
Kanwal Deep Kaur \\ Department of CSE \\ GGITS, Jabalpur
}

\author{
Preeti Rai, PhD \\ Department of CSE \\ GGITS, Jabalpur
}

\begin{abstract}
There has been a growing interest in automatic age and gender classification, as it has become relevant to an increasing amount of applications such as human-computer interaction, surveillance, biometrics, intelligent marketing and many more. Facial age and gender from the face image of a person is one such significant demographic attribute. In this paper, presents a review of automatic facial gender classification and age estimation framework in computer vision. While highlighting the challenges involved during classification of images captured under unconstrained conditions or may be the laborious process of gathering the face images for age estimation, as aging is the uncontrolled and slow process. A comprehensive survey for facial feature extraction methods and face databases for gender and age estimation studied in the past couple of decades is mentioned. Evaluation and result based performance achieved for various face images from different databases has been explained.
\end{abstract}

\section{General Terms}

Gender classification, Facial-age estimation, Algorithm, Facial image, Face image database

\section{Keywords}

Feature extraction, Face recognition, Pre-processing, Aging Pattern, Dimension reduction, Geometric based, Appearance based.

\section{INTRODUCTION}

Human face provides most important visual information that can reveal a wide variety of information, whether identity, age, gender, race and etc. These basic attributes like age and gender play fundamental roles in our day to day lives. Facial information differs from person to person, still human can determine the gender and age of the person just by a simple inspection of their face, on the other hand to accomplish the same task computationally by analysis of human facial image is a challenging one for computer system. As it requires extraction of distinct features and attributes from the persons face image to classify them as 'male' or 'female' of age group as 'child', 'teenage', 'mid-age' or 'senior-citizen'. Thus, enabling a computer system to discriminate the face images on the basis of gender and age of the person is yet to be a challenging task.

There has been a growing interest in automatic estimation and classification of the demographic information from the human face images. Automated gender classification and age estimation has many important applications, for example visual surveillance, intelligent user interface, collecting statics for marketing, access control and law enforcement. The ability to retrieve information accurately and reliably from facial depth images has many more practical applications.
Classification of the face images based on the gender and age of the persons face image has received much research in last two decades. Past approaches to estimate or classify these attributes from face image have been relied on differences in facial feature dimensions or tailored face descriptors. Many of them have established classification schemes designed particularly for age or gender estimation tasks. Few of them were designed for the real-life faces acquired in unconstrained imaging conditions or for un-filtered faces and occluded faces.

This paper simply presents a comprehensive review of the methods that have been used for gender classification and age estimation based on the facial mage of a person, with emphasis on feature extraction methods on various bench marks which outperform current state-of-art. In section 2 brief of the research undertaken in the area of gender classification along with the facial age estimation has been mentioned. The system framework and various challenges the are described in section 3. Due to the importance of the feature extraction to the performance of the system, section 4 is dedicated to a comprehensive review of various feature extraction techniques. Section 5 deals with some of the databases used for the evaluation and benchmarking of gender classification and age estimation. In section 6 , tabulated summary on results and performances obtained from previous research works have been described. Finally, the conclusions are drawn in section 7 .

\section{LITERATURE REVIEW}

This section describes an extensive review of the research undertaken in the domain related to face recognition along with the gender classification and facial age estimation from the face image.

A new age estimation approach considering the intrinsic factors of human ages has been proposed by Wei-Lun, JunZua and Jian-Jiun [1]. They presented an age-oriented local regression algorithm called KNN-SVR to capture the complicated facial aging process over the most widely used FG-NET aging database. The proposed approach achieves the lowest mean absolute error (MAE) against the state-of-art algorithms under several experimental settings.

Eran, Enbar and Hassner [2] presented a unique dataset of face images, labelled with age and gender, acquired by smartphones and other mobile devices, and uploaded them to online image repositories without manual filtering in order to show the most challenging image collection compared with other face image benchmarks. And described a robust face alignment technique 'dropout-SVM' which explicitly considers the uncertainties of face feature detectors.

Estimation of gender on real life faces acquired under unconstrained conditions proposed by Caifeng Shan [3], 
describes that classification rate of $94.81 \%$ can be obtained on the LFW database. For such achievement, apply discriminative LBP-Histogram bins as compact facial representation, by adopting SVM with the selected LBPH bins.

Mohamed Abdou Berbar [4] proposed two methods using DCT to extract facial features based on division of image into $\mathrm{m} \times \mathrm{m}$ sub images then applying DCT on each sub image. In first method DCT1, the feature vector consisting first row, the first column, and the diagonal of DCT coefficients were calculated from each sub image. In second method DCT2, feature vector formed by the concatenation of average values of nonoverlapping $\mathrm{n} \times \mathrm{n}$ square arears of the DCT coefficients of each sub image. The second approach was based on using gray-level co-occurrence matrix (GLCM), to extract texture features. The third approach was based on wavelet transform for facial features extraction. For precise evaluation, the databases used are AT@T, FERET, UMIST \& Sheffield and Faces94.The accuracy results from using texture feature extractions from GLCM for all databases were excellent accuracies and competing DCT2. With the use of 2D-WT accuracies for all database were ranging between $96.18 \%$ and $99.6 \%$ (except FERET, its accuracy was $92 \%$ ).

Human faces captured in real world conditions contains large variation in shape and occlusions due to differences in pose, expression, or any accessories such as sunglasses, scarf or interaction with food or mobile phone. Thus, some estimation approaches struggle under such conditions since they fail to provide a principled way of handling outliers. Therefore, Xavier P. Perona and Piotr Doll [5] together proposed a novel method called Robust Cascaded Pose Regression(RCPR) which reduces exposure to outliers by detecting occlusions explicitly and used robust shape-indexed features.

Demographic estimation from face images: Human versus Machine performance has been proposed by $\mathrm{Hu}$ Han, Charles Otto, and Anil K. Jain [6][7]. They proposed a hierarchical approach for automatic age, gender and race estimation and provided an analysis for how long aging influence, individual facial components. Experimental results on diverse set of face image databases were discussed.

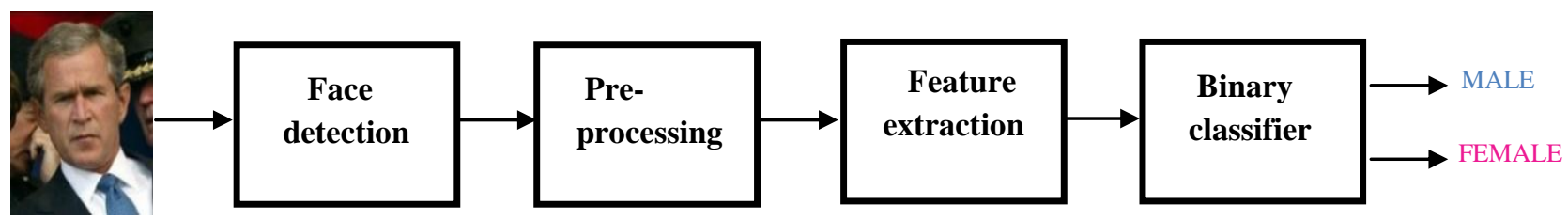

Figure 1 Typical Framework for Gender Classification System

Juan Bekios-Calfa, Jose m.Buenaposada and Luis Baumela [8], studied the problem of gender recognition from a multiattribute perspective. Gender recognition under constrained conditions (e.g. the color FERET database) is a well-known problem for which statement of the art algorithms provide performances well above 90\%. However, when these algorithms are tested under real life conditions, significant drop in performance can be seen. The existence of conditional dependencies among various attributes like; gender, age and pose facial attributes, proves improvements in the performance of gender classifier by exploiting these relations. They achieved an $80.53 \%$ success rate for the real setting in GROUPS database using an appearance based multi attribute linear classifier.

\section{SYSTEM FRAMEWORKS AND CHALLENGES}

In this section, a precise description of various framework and challenges of a gender classification and an age estimation recognition system has been mentioned.

\subsection{Gender Classification Framework}

Among the sizable literature available on the gender classification from the face image, here is a general framework of the system. Generally, a classification system consists of face detection, pre-processing, feature extraction and binary classifier. But, in frameworks like neural networks, feature extraction and classification have been integrated as a single module [17].

The typical diagram for the gender classification system has been shown in Figure 1. The dedicated system first will fetch the object image (here its face image) from the dataset, (the collection of sample images is known as dataset) and the preprocessing module gives the pre-processed image by extraction of only the relevant face area from the whole image. Now, the feature extraction module will extract the important distinguishing features or attributes and store them as 'stored feature' and these features will be used by the binary classifier to classify the subject as either 'male' or 'female' [7] [10].

Generally, a face detection is concerned with finding faces in any given images and, if present, return the image location and content of each image [19]. Mostly, the images available in face databases contains some irrelevant details from the classification point of view. Thus, the pre-processing procedure applied over the image may include the following.

$>$ The face image dataset may available in the RGB form, so it should get converted into gray-scale image.

$>$ The region comprising of only face information is

$>$ Termed as the 'relevant area of interest' or 'region of interest'; simply 'ROI' and it should be cropped.

> Removal of regions external to the face, such as hair, neck or any accessories (like earring, scarfs and etc.)

$>$ Downsizing to reduce the number of pixels (e.g. by using wavelet filters)

$>$ Normalize for contrast and brightness (e.g. using histogram equalization)

$>$ Rescaling or normalizing the pixel values, for example to zero mean and unit variance.

$>$ Reduction of size of image without losing its potential data. (e.g. by using DWT)

The example of above mentioned pre-processing steps has been shown in figure 2 .

Now in feature extraction, selection of the most discriminative features has been done, when the feature number are so large, 
then dimension reduction techniques such as LDA (Linear Discriminant Analysis) and PCA (Principal Component Analysis) can be applied. To enhance the performance fusion of features from different methods can also be applied. Some of the feature extraction methods has been discussed at details in section 4 .

The last mile in the gender classification system is use of classifier that is classification step. In this the subject has to be classified as either male or female. Thus, to achieve this a binary classifier is used. For the supervised learning approach technique, the binary classifier is first trained with some sample images for each class (here, it is male and female) and after that its evaluated with the set of images. The example of the binary classifiers for gender classification from the literature history, that have been deployed are support vector machine (SVM), k- nearest neighbours (k-NN), Bayesian classifier, Adaboost, neural networks.

Based on the review, SVM has found to better classifier as compared with Adaboost and k-NN classifiers.

\subsection{Age Estimation Framework}

There were several age estimation algorithms published in the last decade, these algorithms can be separated into two categories [1]: First is to estimate the actual age (for e.g. 20year old); and the second is to classify

a person image into an age range, like a baby, teenage, middle-age or a senior person. An age estimation system can be simply divided into three steps: image input, feature extraction and age estimation or age determination. The typical age estimation system diagram is shown in figure 3 .

For facial feature related to human ages or facial appearance change are extracted from human faces for compact representation; afterwards in an age estimation function is built to estimate the age based on the extracted features.

If considered an age estimation as a conventional classification problem [23], then the simplest way is to model face images at each age. Researchers found out that; 'Age' is a relative concept specified to each person; every different person age in different ways [20] [21] [23]. A face at particular age is more related to the same persons face at different age rather than to other persons face at different age. Thus, they prepared an aging pattern, the concept of aging pattern can be described as an aging pattern is a sequence of personal face images sorted in time order. Figure 4 shows some typical examples of the "full-filled" aging patterns when AGES (Aging pattern Subspace, an algorithm for automatic age estimation) [23] is applied over FG-NET Aging database.

\subsection{Challenges}

Face images captured in unconstrained or real-world conditions contains large number of variations in terms of shape and occlusions due to differences in pose, expressions (neutral, smiling, closed eyes etc.), partial occlusion of the faces such as, use of any accessories such as sunglasses, scarfs, hats due to weather conditions or ear rings and interactions with objects (e.g. food or cell phone) or facial hair

[5]. Factors due to the image captusre process are the person's head pose, lighting or illumination and image quality issues like blurring, low resolution and noise present in the image [17].

These variations pose a big challenge to the capability of a face recognitions process, and therefore system may get fail to provide a principled way of handling the situation.
The facial appearance changing rates at different aging stages are different; usually, the young faces changes faster as compared with the older one. Therefore, age estimation can be more susceptible for causing error in the older ages. The phenomenon is known as Imbalanced Age Estimation [21]. Likewise, sometimes the identification of gender for small children, babies or infants can be a big task as both male or female looks alike.

The challenges in an automatic age estimation is mainly due to aging effects on the face when it compared with other face variations. The following points includes unique characteristics of aging variation:

$>$ The aging process is uncontrollable; since the procedure of aging is slow ad irreversible.

$>$ Consequently, the collection of sufficient training data for age estimation is extremely laborious.

$>$ Every different person has their personalized ageing patterns, and it is determined by their gene as well as many external factors, such as health, living style and weather conditions etc. [23]

Figure 5 and 6 shows some example of the above conditions which can cause the challenging situations while face recognition [7] [14].

\section{FEATURE EXTRACTION METHODS}

Feature extraction is the selection information required to describe a large set of data. Feature extraction methods for face gender classification and age estimation can be broadly classified into two parts; that are geometric based and appearance based approaches [17].

\subsection{Geometric based Approach}

This approach is based on measurements of facial landmarks. These landmarks are important points on the face that mark its features. In this approach, geometric relationships between these points are maintained but other possibly useful information is neglected. Furthermore, accuracy is required in the process of extracting the point locations.

\subsection{Appearance based Approach}

These methods are based on some operation or transformation performed on the pixels of an image. This can be done at the global or local level. At the global level, features are computed from the whole image resulting in a single feature vector. In local feature extraction, the image is partitioned beforehand into some arbitrary regions (which may be equally spaced or otherwise) or into semantically meaningful regions such as eyes, nose and mouth areas. A feature vector is then obtained from each patch.
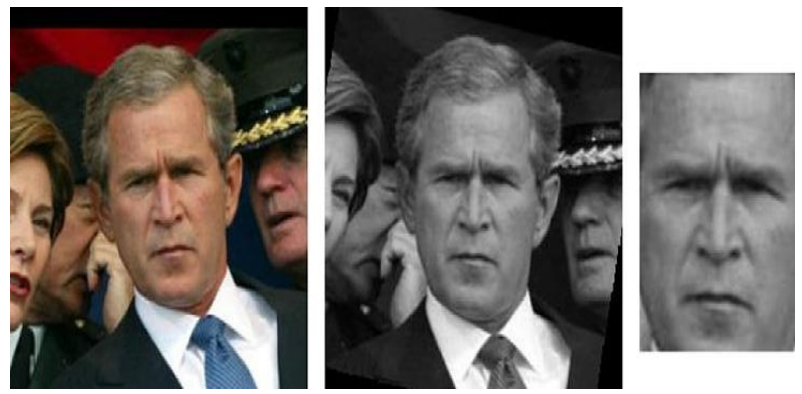

Figure 2. The pre-processing process on face images. (left) original image; (middle) aligned image; (right) cropped face 
In the following subsection; Table 1 gives summarized description of feature extraction methods.

\section{DATABASE}

There exist several publicly available databases that has been used for the purpose of evaluating gender classification and age estimation approach. For training and evaluation of their proposed approaches, some researchers take only a subset (dataset) of the databases (for e.g. like taking only frontal images), or to obtain a huge number of images, they combine two or more datasets. For absence of data they desired to have, they use their own dataset, by offering a unique dataset of face images acquired by smart phones, obtained from inter-net, and may also labelled them with age and gender, thus making the collection more challenging.

Another common practice in data collection is to use a face detector to obtain cropped faces from images of people. However, this may cause the data to be biased, for example if the detector successfully detects only frontal and near frontal faces.

Some of the publicly available face image database are

\section{summarized in Table 2}

The number of images and the number of unique individuals in each dataset are shown, to the best of our knowledge.

\section{EVALUATION AND RESULT}

This section will represent results for some face image gender classification and age estimation experiments performed by the researchers in literature. Consequently, FERET is known as the most widely used dataset for evaluating gender [17] recognition methods, and for age estimation FG-NET and MORPH [13] has been widely used. Table 3 shows the summary on performance achieved on gender classification, which is generally measured as CCR. Correct Classification Rate (CCR) is the percentage of the ratio of correctly classified images with total number of images present in the dataset. Further, the performance of age estimation system in different situation segregated into three different cases, which is usually measured by the Mean Absolute Error (MAE) [6][7] [22] [23], defined as the average of the absolute errors between ages and the ground truth ages.

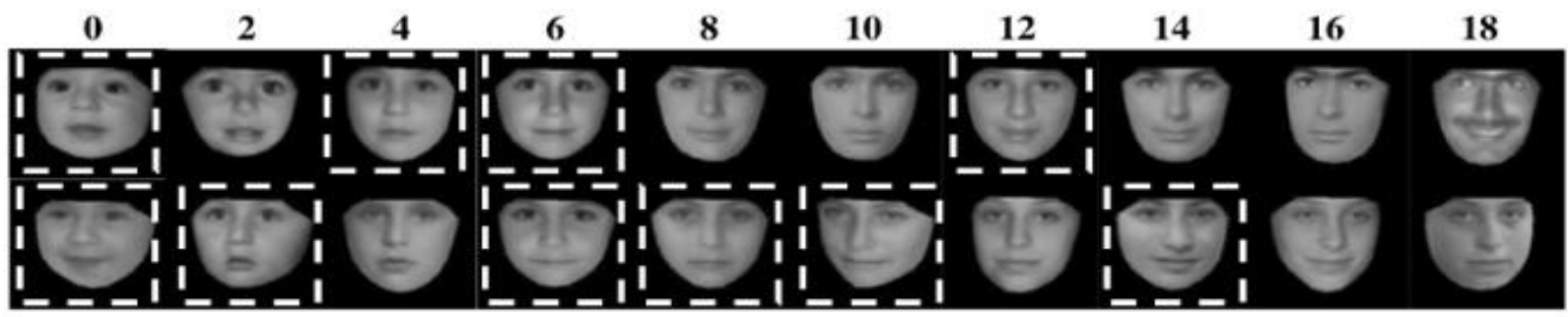

Figure 4. The full filled aging pattern. Each line shows the aging pattern of one person. The ages are marked above the corresponding faces. The faces learned by the algorithms are surrounded by the dashed line squares. [23].

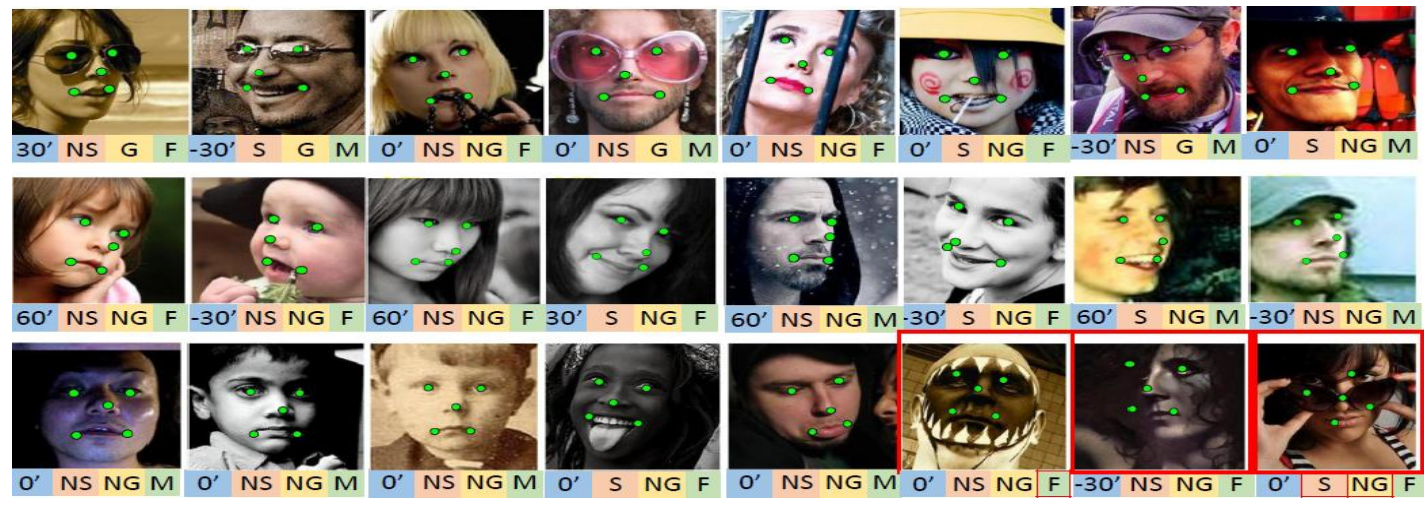

Figure 5. Results on AFLW: Faces with occlusion (row 1), pose variation (row 2), different lighting conditions (column 1-2 in row 3), low image quality (column 3 in row 3), different expressions (column 4-5 in row 3), three inaccurate cases are shown in column 6-8 in row $3 .[14]$

Table 1. Review of Some Feature Extraction Methods

\begin{tabular}{|l|l|}
\hline Feature Extraction method & Summary \\
\hline $\begin{array}{l}\text { Principal Component } \\
\text { Analysis }\end{array}$ & $\begin{array}{l}\text { PCA is mathematically defined as an orthogonal linear transformation that } \\
\text { transforms the data to a new coordinate system such that the greatest variance by } \\
\text { some projection of the data comes to lie on the first coordinate (called the first } \\
\text { principal component), the second greatest variance on the second coordinate, and so } \\
\text { on. }\end{array}$ \\
$\begin{array}{l}\text { For example, consider a data matrix, } \mathbf{X} \text {, with column-wise zero empirical mean (the } \\
\text { ample mean of each column has been shifted to zero), where each of the } n \text { rows } \\
\text { represents a different repetition of the experiment, and each of the } p \text { columns gives a } \\
\text { particular kind of feature (say, the results from a particular sensor). }\end{array}$ \\
\hline
\end{tabular}




\begin{tabular}{|c|c|}
\hline $\begin{array}{l}\text { Multi-Manifold } \\
\text { Discriminant Analysis }\end{array}$ & $\begin{array}{l}\text { In MMDA, the within-class graph and between-class graph are designed, } \\
\text { respectively to characterize the within-class compactness and the between- class } \\
\text { separability, and define the criterion function to calculate projection matrix, seeking } \\
\text { for the discriminant matrix to simultaneously maximize the between-class scatter and } \\
\text { minimize the within-class scatter. Thus, } \\
\text { the within-class graph can represent the sub-manifold information, while between- } \\
\text { class graph can represent the multi manifold information }\end{array}$ \\
\hline Local Binary Patterns & $\begin{array}{l}\text { In LBP, LBP feature vector is created in its simplest form by using following steps: } \\
\text { - Examine window id divided into cells (e.g. } 20 \mathrm{X} 20 \text { pixels for each cell.) } \\
\text { - Comparison for each pixel in a cell to each its } 8 \text { neighbors (i.e. on its right- } \\
\text { top, left-top, right-bottom, left-middle and etc.), along a circle in clockwise } \\
\text { or counter clockwise direction. } \\
\text { A } 8 \text {-digit binary number is generated (which is usually converted into } \\
\text { decimal for convenience) by putting " } 0 \text { " where the center pixels value is } \\
\text { greater than the neighbors value else put " } 1 \text { ". } \\
\text { - Now, histogram is computed over the cell, of the frequency of each number } \\
\text { occurring. This histogram can be seen as a } 256 \text {-dimensional feature vector. } \\
\text { - Normalization of the histogram, which is the optional step. } \\
\text { - Concatenation of histograms(normalized) of all cell, gives a feature vector } \\
\text { - The feature vector can now be processed using any classifier. }\end{array}$ \\
\hline Gabor & $\begin{array}{l}\text { In the fields of computer vision, pattern recognition and image processing, gabor } \\
\text { filter has large number of applications [55]. 2D Gabor filter is a selective filter in } \\
\text { terms of frequency and orientation. Gabor filter response hasn't been disturbed by } \\
\text { noise and distortion exists at different locations due to accuracy in time-frequency } \\
\text { localization. Hence, performance of gabor filter is upto mark for noisy images [56]. } \\
\text { As modulated by Gaussian envelop [57], for particular frequency and orientation, } \\
\text { gabor filter is being considered as a sinusoidal plane. } \\
\mathrm{hx}, \mathrm{y}=\mathrm{s}(\mathrm{x}, \mathrm{y}) \times \mathrm{g}(\mathrm{x}, \mathrm{y}) \\
\text { Where, } \mathrm{s}(\mathrm{x}, \mathrm{y}) \text { is a sinusoidal plane of particular frequency and orientation; and } \mathrm{g}(\mathrm{x} \text {, } \\
\mathrm{y}) \text { is a } \\
\text { 2D Gaussian function known as envelop. }\end{array}$ \\
\hline Discreate Cosine Transform & $\begin{array}{l}\text { A DCT expresses a finite sequence of data points in terms of a cosine functions } \\
\text { oscillating at different frequencies, while small high-frequency components can be } \\
\text { discarded. The DCT is a Linear invertible function or equivalently an invertible } \mathrm{N} X \\
\mathrm{~N} \text { square matrix. There are several variants of the DCT with slightly modified } \\
\text { definitions. The } N \text { real numbers } x_{0}, \ldots, x_{N-1} \text { are transformed into the } N \text { real } \\
\text { numbers } X_{0}, \ldots, X_{N-1} \text {. }\end{array}$ \\
\hline $\begin{array}{l}\text { Scale invariant feature } \\
\text { transform }\end{array}$ & $\begin{array}{l}\text { SIFT extracts feature descriptors from various key points in an image. } \\
\text { The key points are detected from the scale-space extrema, which typically } \\
\text { correspond to edges, corners and other informative structural changes in the image. } \\
\text { The descriptors are formed by the orientation histograms of gradient directions over } \\
\text { local regions around the key point. SIFT features are invariant to image scaling and } \\
\text { rotation, and partially invariant to illumination changes and affine distortions. Using } \\
\text { these descriptors, objects can be reliably recognized even from different views or }\end{array}$ \\
\hline
\end{tabular}




\begin{tabular}{|c|c|}
\hline & under occlusion. \\
\hline Pixel intensity values & $\begin{array}{l}\text { Pixel intensity values can be used directly as input to train a classifier such as in } \\
\text { neural network or SVM. The images (after cropping the head), are usually } \\
\text { normalized as a pre-processing step, to compensate for geometric and lighting } \\
\text { variations, and then down-sampled to smaller sizes. }\end{array}$ \\
\hline 2 Dimensional PCA & $\begin{array}{l}\text { 2DPCA is based on 2D image matrices rather than } 1 \mathrm{D} \text { vectors so the image matrix } \\
\text { does not need to be transformed into a vector prior to feature extraction. Instead, an } \\
\text { image covariance matrix is constructed directly using the original image matrices, } \\
\text { and its eigenvectors are derived for image feature extraction. }\end{array}$ \\
\hline Local Preserving Projections & $\begin{array}{l}\text { LPP is a well-known method for image feature extraction and dimension reduction. } \\
\text { The objective of an LPP is to preserve the local structure of the image space by } \\
\text { explicitly considering the manifold structure. }\end{array}$ \\
\hline $\begin{array}{l}\text { Linear Discriminant } \\
\text { Analysis }\end{array}$ & 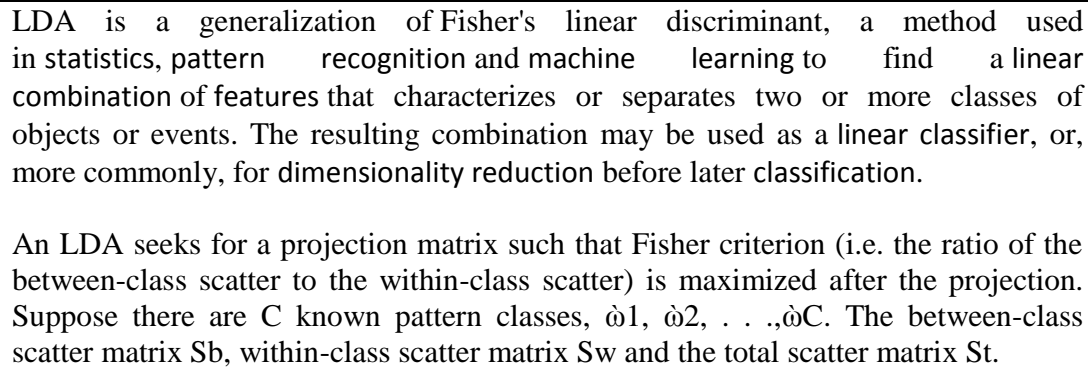 \\
\hline
\end{tabular}

Recently proposed manifold learning methods, called marginal fisher analysis (MFA) and locality sensitive discriminant analysis [11] [22], for the age estimation problem. OLPP with biologically inspired features(BIF) has been applied for age estimation rather than raw image, a significant gain in performance has been obtained. Along with Principal component analysis (PCA), the four different face representations: 'BIF + PCA', 'BIF + OLPP, 'BIF + LSDA and 'BIF + MFA are compared

This paper presents a comprehensive review on facial gender classification and age estimation using computer vision-based methods, focusing on 2-D approaches. A general framework of both the systems was presented and discussed. Also highlighted the challenges, as well as provided a detailed review of the state-of-the-art methods and commonly used features.

Table 2. Publicly available Face Databases

\begin{tabular}{|l|l|l|l|l|l|}
\hline Dataset & $\begin{array}{l}\text { No. of } \\
\text { images }\end{array}$ & $\begin{array}{l}\text { No. of } \\
\text { unique } \\
\text { individual } \\
\text { s }\end{array}$ & $\begin{array}{l}\text { Ge } \\
\text { nde } \\
\mathbf{r} \\
\text { lab } \\
\text { els }\end{array}$ & $\begin{array}{l}\text { Ag } \\
\text { lab } \\
\text { els }\end{array}$ & $\begin{array}{l}\text { Controll } \\
\text { ed } \\
\text { variatio } \\
\text { ns }\end{array}$ \\
\hline $\begin{array}{l}\text { FERET } \\
{[7]}\end{array}$ & 14126 & 1199 & No & No & P, L, X \\
\hline FEI & 2800 & $\begin{array}{l}200(100 \mathrm{~m}, \\
100 f)\end{array}$ & & & \\
\hline AR & $<4000$ & $\begin{array}{l}126(70 \mathrm{~m}, \\
56 \mathrm{f})\end{array}$ & Yes & & X, L, O \\
\hline $\begin{array}{l}\text { LFW } \\
{[7][8]}\end{array}$ & $13233(1025$ \\
$6 \mathrm{~m}, 2977 \mathrm{f})$ & 5749 & No & & $\begin{array}{l}\text { Uncontro } \\
\text { lled }\end{array}$ \\
\hline $\begin{array}{l}\text { Adience } \\
{[15]}\end{array}$ & 26000 & 2284 & & & $\begin{array}{l}\text { Uncontro } \\
\text { lled }\end{array}$ \\
\hline PAL [6] & 844 & 590 & & & \\
\hline
\end{tabular}




\begin{tabular}{|c|c|c|c|c|c|}
\hline $\begin{array}{l}\text { MULTI } \\
\text { PIE }\end{array}$ & $\begin{array}{l}\text { More than } \\
750000\end{array}$ & 337 & & & $\mathrm{P}, \mathrm{L}$ \\
\hline YGA [7] & 8000 & 1600 & & & \\
\hline $\begin{array}{l}\text { AT\&T } \\
\text { [4] or } \\
\text { ORL }\end{array}$ & 4000 & 40 & & & $\mathrm{P}, \mathrm{L}$ \\
\hline $\begin{array}{l}\text { UMIST } \\
{[4]}\end{array}$ & 564 & 20 & & & $\mathrm{P}$ \\
\hline $\begin{array}{l}\text { LFPW } \\
\text { [5] }\end{array}$ & 1300 & 29 & & & $\begin{array}{l}\text { Uncontro } \\
\text { lled }\end{array}$ \\
\hline $\begin{array}{l}\text { HELEN } \\
{[5]}\end{array}$ & 2330 & 194 & & & \\
\hline $\begin{array}{l}\text { PCSO } \\
{[6]}\end{array}$ & 1.5 million & & & & \\
\hline $\begin{array}{l}\text { FG-NET } \\
\text { [6] [23] }\end{array}$ & 1002 & 82 & & & \\
\hline $\begin{array}{l}\text { GROUP } \\
\text { S [8] }\end{array}$ & 23218 & 1881 & Yes & No & $\begin{array}{l}\text { Uncontro } \\
\text { lled }\end{array}$ \\
\hline $\begin{array}{l}\text { MORPH } \\
{[8]}\end{array}$ & $\begin{array}{l}55285(4676 \\
7 \mathrm{~m}, 8518 \mathrm{f})\end{array}$ & 13660 & Yes & $\begin{array}{l}\text { Ye } \\
\mathrm{s}\end{array}$ & Age \\
\hline $\begin{array}{l}\text { Yale } \\
\text { Face } \\
\text { Databas } \\
\text { e B }\end{array}$ & 16128 & 28 & No & No & $\mathrm{P}, \mathrm{L}$ \\
\hline $\begin{array}{l}\text { INDIAN } \\
\text { FACE }\end{array}$ & 4400 & 40 & & & \\
\hline \multicolumn{6}{|c|}{$\begin{array}{l}P \text { pose or view, L lighting or illumination, } X \text { expression, } O \\
\text { occlusion }\end{array}$} \\
\hline
\end{tabular}

Feature extraction for facial recognition can be categorized into geometric-based and appearance-based methods, further some of the feature extraction methods are summarized in a tabular form followed by that classifier can be used to achieved the desired performance.

Some of the important datasets for evaluating gender classifiers and age estimation system were described. Evaluation and representative results were reviewed.

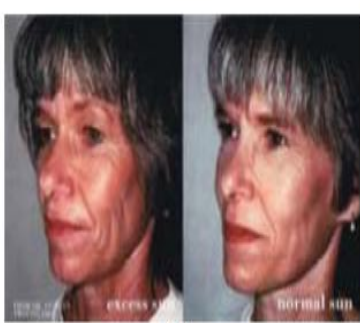

(a)

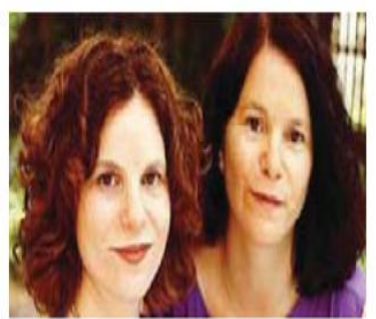

(b)
Figure 6. Different facial appearances of identical twins possibly due to extrinsic factors such as (a) environmental conditions (e.g. sunshine), and (b) lifestyle. [7]
Table 3. A Summary on Performance of the Existing Work on Gender Classification

\begin{tabular}{|c|c|c|c|}
\hline $\begin{array}{l}\text { Existing } \\
\text { Works }\end{array}$ & $\begin{array}{l}\text { Feature } \\
\text { Extraction } \\
\text { Method } \\
\text { Approach }\end{array}$ & Database & $\begin{array}{l}\text { Correct } \\
\text { Classification } \\
\text { Rate (in } \\
\text { percent) }\end{array}$ \\
\hline $\begin{array}{l}\text { E. } \\
\text { Eidinger } \\
{[2]}\end{array}$ & $\begin{array}{l}\text { LBP and } \\
\text { FPLBP }\end{array}$ & $\begin{array}{l}\text { Provided a } \\
\text { unique own } \\
\text { dataset of faces } \\
\text { labelled with } \\
\text { age and gender }\end{array}$ & $75 \%$ to $87.5 \%$ \\
\hline $\begin{array}{l}\text { S. Caifeng } \\
\text { [3] }\end{array}$ & LBPH bins & LFW & $94.81 \%$ \\
\hline $\begin{array}{l}\text { Mohamed } \\
\text { A Berbar } \\
\text { [4] }\end{array}$ & $\begin{array}{l}\text { DCT, GLCM, } \\
\text { DWT }\end{array}$ & $\begin{array}{l}\text { AT@T, } \\
\text { FERET, } \\
\text { UMIST, } \\
\text { FACES94 }\end{array}$ & $\begin{array}{l}96.18 \% \text { to } \\
99.6 \% \text { except } \\
\text { for } \\
\text { FERET }(92 \%)\end{array}$ \\
\hline $\begin{array}{l}\text { Xavier P. } \\
\text { Burgos- } \\
\text { Artizzu [5] }\end{array}$ & $\begin{array}{ll}\text { Robust face } \\
\text { landmark } \\
\text { estimation } \\
\text { under }\end{array}$ & $\begin{array}{l}\text { LFPW, LFW } \\
\text { and HELEN }\end{array}$ & $\begin{array}{l}\text { detects } \\
\text { occlusion } \\
\text { with }\end{array}$ \\
\hline
\end{tabular}




\begin{tabular}{|c|c|c|c|}
\hline & $\begin{array}{l}\text { occlusion using } \\
\text { RCPR }\end{array}$ & & $\begin{array}{l}80 / 40 \% \\
\text { precision/recall }\end{array}$ \\
\hline H. Han [7] & $\begin{array}{l}\text { predicted the } \\
\text { demographic } \\
\text { attributes of the } \\
\text { face image by } \\
\text { using } \\
\text { hierarchical a } \\
\text { classifier and } \\
\text { prepared C++ } \\
\text { implementation } \\
\text { of such } \\
\text { algorithm. }\end{array}$ & $\begin{array}{l}\text { FG-NET, } \\
\text { FERET, } \\
\text { MORPH } \\
\text { Album2, PCSO } \\
\text { and LFW }\end{array}$ & $93 \%$ to $97.6 \%$ \\
\hline $\begin{array}{l}\text { Juan } \\
\text { Bekios- } \\
\text { Calfa [8] }\end{array}$ & $\begin{array}{l}\text { PCA, } \\
\text { LDA and SDA }\end{array}$ & Color FERET & Above $90 \%$ \\
\hline $\begin{array}{l}\text { Juan E. } \\
\text { Tapia, and } \\
\text { Claudio A. } \\
\text { Perez [9] }\end{array}$ & $\begin{array}{l}\text { LBP } \quad \text { and } \\
\text { LBPH }\end{array}$ & $\begin{array}{l}\text { FERET, UND, } \\
\text { LFW and AR }\end{array}$ & $\begin{array}{l}70 \% \text { on the } \\
\text { FERET } \\
\text { database, } \\
73 \% \text { on the } \\
\text { UND and } 90 \% \\
\text { on the LFW } \\
\text { database }\end{array}$ \\
\hline
\end{tabular}

\section{REFERENCES}

[1] Wei-Lun Chao n, Jun-Zuo Liu and Jian-Jiun Ding, Vol 46, Issue 3, March 2013. Facial age estimation based on label-sensitive learning and age-oriented regression.

[2] Eran Eidinger, Roee Enbar and Tal Hassner, Vol 9, no.12, pp.2170-2179, December 2014. Age and Gender Estimation of Unfiltered Faces.

[3] Caifeng Shan, Pattern Recognition Letters, Vol 33, no. 4, pp.431-437, March 2012. Learning local binary patterns for gender classification on real world face images.

[4] Mohamed Abdou Berbar, Springer-The Visual Computer, Vol 30, no. 1, pp.19-31, January 2014. Three robust features extraction approaches for facial gender classification.

[5] Xavier P. Burgos-Artizzu, Pietro Perona and Piotr Doll'ar, IEEE explorer 2013. Robust face landmark estimation under occlusion.

[6] Hu Han, Charles Otto, and Anil K. Jain, Vol. 37, No. 6, pp. 1148-1161, June 2015. Age Estimation from Face Images: Human vs. Machine Performance.

[7] Hu Han, Charles Otto, Xiaoming Liu and Anil K. Jain, IEEE transactions on Pattern analysis and Machine intelligence, Vol 37, no. 6, pp.1148-1161, October 2014. Demographic Estimation from Face Images:Human vs. Machine Performance.

[8] Juan Bekios-Calfa, Jose m. Buenaposada and Luis Baumela, Vol 36, pp 228-234. Pattern Recognition Letters 2013. Robust gender recognition by exploting facial attributes dependencies.

[9] Juan E. Tapia, and Claudio A. Perez, IEEE transactions on information forensics and security, vol. 8, no. 3, MARCH 2013. Gender Classification Based on Fusion of Different Spatial Scale Features Selected by Mutual Information From Histogram of LBP, Intensity, and Shape.

[10] P. Rai and P. Khanna, Journal of Visual Communication and Image Representation. 25, no. 5, pp. 1118-1129, 2014.A gender classification system robust to occlusion using gabor features based (2d)2pca.

[11] Wankou Yang, Changyin Sun and Lei Zhang, In Pattern Recognition, www.elsevier.com,sciencedirect, 2011. A multi manifold discriminant analysis method for image feature extraction.

[12] Xin Geng, Kate Smith-Miles, Zhi-Hua Zhou, Vol 35, Issue10, Oct. 2013. Facial Age Estimation by Learning from Label Distributions.

[13] Ke Chen, Shaogang Gong, Tao Xiang and Chen Change Loy, The IEEE Conference on Computer Vision and Pattern Recognition (CVPR), 2013, pp. 24672474.Cumulative Attribute Space for Age and Crowd Density Estimation.

[14] Zhanpeng Zhang, Ping Luo, Chen Change Loy, and Xiaoou Tang, Springer pp 94-108, 2014Facial Landmark Detection by Deep Multi-task Learning.

[15] Gil Levi and Tal Hassner, Computer vision foundation, IEEE explorer 2015. Age and Gender Classification using Convolutional Neural Networks.

[16] Giancarlo Taveira and Leandro A.F. Fernandes, Pattern recognition letters Volume 50, 1 December 2014, Pages 82-90. Automatic alignment and reconstruction of facial depth images

[17] Choon-Boon Ng, Yong-Haur Tay and Bok-Min Goi, Pattern Analysis and Applications November 2015, Volume 18, Issue 4, pp 739-755 ,A review of facial gender recognition.

[18] Ning Zhang, Manohar Paluri, Marc'Aurelio Ranzato, Trevor Darrell and Lubomir Bourdev, Computer Vision Foundation., IEEE explorer, 2014. PANDA: Pose Aligned Networks for Deep Attribute Modeling.

[19] Ming-Hsuan Yang, Researchgate, January 2015 Face Detection.

[20] Lanitis, Draganova and Christodoulou, IEEE transactions pp 621-628, Vol 34, issue 1, January 2014, Comparing Different Classifiers for Automatic Age Estimation.

[21] Xin Geng, Zhi-Hua Zhou and Kate Smith-Miles, IEEE transactions on pattern analysis and machine intelligence, vol. 29, no. 12, December 2007, Automatic Age Estimation Based on Facial Aging Patterns.

[22] Guodong Guo, Guowang Mu, Yun Fu, Charles Dyer and Thomas Huang, Computer Vision, IEEE explorer,2009. A Study on Automatic Age Estimation using a Large Database.

[23] Xin Geng, Zhi-Hua Zhou, Yu Zhang, Gang Li, and Honghua Dai, ACM international conference on Multimedia, Pages 307-316 October, 2006. Learning from Facial Aging Patterns for Automatic Age Estimation. 